Ann. Zootech., I980, 29 (2), I2I-I36.

\title{
Influence de l'apport protéique durant la croissance et la première gestation sur les performances de reproduction et la composition corporelle chez la truie
}

\author{
P. H. DUEE, F. TREIL et Sylvaine CAMOUS
}

Avec la collaboration technique de Michèle SEREZAT, G. CONSEIL et J. LEBOST

Station de Recherches sur l'élevage des porcs

Centre National de Recherches Zootechniques, I.N.R.A.. 78350 Jouy-en-Josas (France)

\section{Résumé}

Une expérience a été entreprise sur 120 porcs femelles de race Lavge White, à partir d'un poids moyen de $28 \mathrm{~kg}$ de poids vif et jusqu'au terme de la première gestation. Les animaux sont répartis, jusqu'à la puberté, en 3 lots ne différant que par le taux en matières azotées du régime distribué. A un lot témoin (lot I : i 6 p. Ioo de matières azotées jusqu'à $60 \mathrm{~kg}$ de poids vif puis I 4 p. Ioo), on compare l'effet sur les performances de croissance et de reproduction d'urie diminution du taux protéique avant $60 \mathrm{~kg}$ de poids vif (lot $2:$ I $2 \mathrm{p}$. I oo) ou après $60 \mathrm{~kg}$ de poids vif (lot 3 : Io p. Ioo). A la puberté, les truies reçoivent l'un des 2 régimes de la période de finition ( 4 ou Io p. Ioo de protéines) au même niveau alimentaire ( $2 \mathrm{~kg}$ par jour jusqu'à $3^{\circ}$ jours puis $\mathrm{I}, 8 \mathrm{~kg}$ ).

La moitié de l'effectif dans chacun des six groupes ainsi constitués est abattue vers $3^{\circ}$ jours de gestation; l'autre moitié vers Io5 jours. La restriction protéique appliquée pendant une partie de la croissance des animaux diminue leur vitesse de croissance (entre $28 \mathrm{~kg}$ de poids vif et la puberté) de 8 p. I oo (lot 2) à I I p. I oo (lot 3) mais le retard non significatif à la puberté n'est que de 9 jours (lot $\mathrm{I}: 240$ jours; lots 2 et $3: 249$ jours), avec, à ce stade, un taux d'ovulation plus faible (lot I : I 3,$8 ; \operatorname{lot} 2:$ I $3, \mathrm{r} ; \operatorname{lot} 3: \mathbf{1} 2,7$ ).

Pour ce qui concerne le nombre d'embryons (à 30 jours) ou de foetus (à ro5 jours), il n'apparaît pas de différence significative entre lots : la mortalité in utéro étant plus élevée dans le lót témoin.

L'influence de 1'apport protéique durant la gestation est ressentie principalement au niveau. maternel. La diminution du taux protéique de $\mathrm{s} 4$ à io p. I oo réduit le gain net de poids des animaux de I 2 p. Ioo, comme la quantité d'azote retenu en fin de gestation (de I 7,0 à $6,4 \mathrm{~g}$ par jour), correspondant à une quantité moindre de masses musculaires déposées.

Toutefois, il semble que l'apport protéique prépubertaire influe sur les modifications de la composition corporelle au cours de la gestation, Une restriction protéique en croissance diminue, au taux de matières azotées le plus bas en gestation, la quantité d'azote retenu et ne permet pas, en général, un đépôt de masses musculaires comparable à celui réalisé dans le lot témoin, thalgré un gain de poids en gestation plus élevé. 


\section{Introduction}

L'apparition de la naturité sexuelle est une composante très variable de la reproduction cher la truie. Si de nombreux facteurs de variation ont été mis en évidence (RERAT et DUEE, I 976), leur effet reste souvent difficile à expliquer et impossible actuellement à mettre en relation avec des différences du profil hormonal des animaux. Parmi ces facteurs, le gain de poids au cours de la croissance joue un rôle non négligeable (CARREz et al., I977).

D'ailleurs, des modifications des apports nutritionnels durant cette période peuvent provoquer des variations sensibles de l'âge au premier œstrus. Ainsi, une restriction sévère ( $45 \mathrm{p}$. I oo du niveau libéral) du niveau alimentaire, à partir de $55 \mathrm{~kg}$ de poids vif, retarde la puberté de deux mois (ETIENNE et DUEE, I973). Ceci semble moins évident avec des restrictions plus modérées (LoDGE et $\mathrm{Mc}$ Pherson, I96I) ou quand seule la consommation protéique est altérée (Jones et Maxweli, I974; DUfis, I977). Un niveau nutritionnel adéquat pendant cette période est cependant requis, si l'on veut maximiser le taux d'ovulation au premier cestrus, et par là même, ne pas diminuer la prolificité à la mise bas.

Une autre question peut également se poser : on sait que, durant la gestation, une réduction quantitative et qualitative des apports est préconisée. Le problème se pose alors de l'opportunité de cette restriction, en regard du changement des apports nutritionnels durant la période prépubertaire. Ce point constitue un des objectifs de l'étude où des modifications de l'apport global de matières azotées ont été appliquées, à partir de $28 \mathrm{~kg}$ de poids vif et jusqu'au terme de la première gestation.

\section{Matériel et méthodes}

L'expérience a été entreprise sur I20 animatux de race Large White, d'un poids moyen initial de $28 \mathrm{~kg}$ à 1'âge moyen de 87 jours et répartis, jusqu'à la puberté, en trois lots ne différant que par le taux en matières azotées du régime distribué. Le choix des différents niveaux d'apport protéique procède des recommandations formulées pour le porc femelle en croissance (HENRY, PION et RERAT, I976). A un lot témoin (lot I), on compare l'effet sur les performances de croissance et de reproduction d'une diminution des quantités de matières azotées ingérées, jusqu'à $60 \mathrm{~kg}$ de poids vif, période dite de croissance (lot 2), ou de $60 \mathrm{~kg}$ de poids vif à la puberté, période dite de finition (lot 3 ). Le niveau de consommation est identique pour tous les animaux et évolue, en fonction de leur poids, par tranche de $5 \mathrm{~kg}$, en augmentant progressivement de 0,2 puis $0, \mathrm{I} \mathrm{kg}$ à partir du niveau initial de $\mathrm{I}, 4 \mathrm{~kg}$ far jour jusqu'à $2,8 \mathrm{~kg}$ par jour, niveau maximum atteint à $80 \mathrm{~kg}$ de poids vif et maintenu jusqu'au premier cestrus détecté.

La maturité sexuelle est détectée, quotidiennement, à partir du moment où les animaux atteignent $70 \mathrm{~kg}$ de poids vif et jusqu'à 300 jours d'âge. A la puberté, les truies sont saillies par un verrat Large White et reçoivent l'un des deux régimes de la période de finition, (I4 ou Io $\mathrm{p}$. Ioo de protéines) au même niveau alimentaire durant leur gestation, à savoir, quotidiennement, $2 \mathrm{~kg}$ jusqu'à 30 jours puis $I, 8 \mathrm{~kg}$, constituant ainsi six groupes d'animaux gravides.

La composition et les résultats d'analyses des différents régimes figurent dans le tableau I. Les animaux sont maintenus en loges de 7 , équipées d'un réfec- 
TABLEAU I

Composition des régimes

Composition of experimental diets

\begin{tabular}{|c|c|c|c|c|}
\hline $\begin{array}{l}\text { Période } \\
\text { Peviod }\end{array}$ & $\begin{array}{c}\text { Avant } 6 \\
\text { Before } 6\end{array}$ & $\begin{array}{l}\text { pords vif } \\
\text { e-wergth }\end{array}$ & $\begin{array}{l}\text { Après } 6 \\
\text { After } 6\end{array}$ & poids vif \\
\hline $\begin{array}{l}\text { Taux protéique (\%) } \\
\text { Crude protein }\end{array}$ & I 6 & $\mathrm{I} / 2$ & I 4 & Io \\
\hline 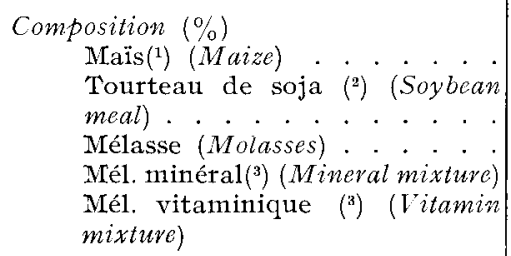 & $\begin{array}{r}73 \\
20 \\
3 \\
3 \\
1\end{array}$ & $\begin{array}{l}85 \\
8 \\
3 \\
3 \\
1\end{array}$ & $\begin{array}{r}80 \\
13 \\
3 \\
3 \\
1\end{array}$ & $\begin{array}{l}88 \\
5 \\
3 \\
3 \\
1\end{array}$ \\
\hline $\begin{array}{l}\text { Resultats d'analyses }(\%) \\
\text { Analytical vesults } \\
\text { Matière sèche }(\text { Dry matter }) . \\
\text { Matières azotées }(\mathrm{N} \times 6,25) \\
\text { (Crude protein). } \\
\text { Energie brute }(\mathrm{Kcal} / \mathrm{kg}) \\
(\text { Ener } g y) . . .\end{array}$ & $\begin{array}{l}89,4 \\
\text { I } 7,3 \\
3910\end{array}$ & $\begin{array}{l}89, \mathrm{I} \\
\mathrm{I} 2,7 \\
387^{\circ}\end{array}$ & $\begin{array}{c}88,7 \\
14,3 \\
3900\end{array}$ & $\begin{array}{l}89,0 \\
\text { I I ,2 } \\
3920\end{array}$ \\
\hline
\end{tabular}

(1) Maïs : 87,3 p. Ioo mat. sèche; 9, I p. roo mat. azotées (Maize: 87.3 p. Ioo dry matter; 9.I p. Ioo crude protein).

(2) Tourteau de soja : 88,7 p. 100 mat. sèche; 50,2 p. roo mat. azotées (Soybean meal: 88.7 p. Ioo dry matter; 50.2 p. I00 crude protein).

$\left({ }^{3}\right)$ DUEE (197\%).

toire individualisé, jusqu'à 30 jours de gestation, puis en loges individuelles pendant le reste de l'expérience.

La moitié de 1'effectif dans chacun des six groupes est abattue entre 27 et 33 jours de gestation et différentes mesures sont effectuées : dénombrement des corps jaunes, nombre et poids moyen des embryous, poids des membranes placentaires. L'autre moitié de l'effectif est maintenue jusqu'à ro5 jours de gestation où le même type de mesures est réalisé.

Par ailleurs, au moment de l'abattage des animaux aux deux stades considérés, on procède à une découpe parisienne des carcasses afint d'apprécier l'évolution de la composition corporelle des animaux au cours de la gestation et en fonction de leur consommation protéique.

Enfin, vers le $80^{e}$ jour de gestation, quatre animaux de chaque groupe sont soumis, après une semaine d'adaptation, à une période de cinq jours en cages de digestibilité pendant laquelle on récolte séparément les fèces et l'urine dans le but de déterminer la digestibilité apparente des matières azotées ingérées et la rétention d'azote.

L'étude statistique des différents paramètres est réalisée au moyen du test d'analyse de variance. 


\section{Résultats}

Par rapport à l'effectif initial de chaque lot, le pourcentage de truies pubères avant 300 jours d'âge apparaît plus faible quand la consommation protéique est restreinte à partir de $60 \mathrm{~kg}$ de poids vif (8I p. roo au lieu de 89 et 90 respectivem ent dans les lots I et 2). De la même façon, le pourcentage de retours en chaleur semble plus élevé avec le niveau le plus bas en protéines durant la gestation (I I,5 p. Ioo contre $7 \mathrm{p}$. Ioo au taux protéique le plus élevé). Il en résulte que l'effectif total des truies gestantes à 30 jours de gestation est de 84 et représente alors 70 p. Ioo de l'effectif initial mis en lot. Cet effectif n'est cependant pas réparti uniformément entre les six groupes d'arimaux et les deux stades d'abattage : le groupe témoin (I6-I4-I4) n'étant que faiblement représenté à 30 jours de gestation.

\section{I. - Performances de croissance des animaux}

I,es restrictions protéiques appliquées durant la période prépubertaire affectent la croissance journalière des animaux (fig. I) et l'indice de consommation, sans que le niveau moyen de consommation, pour chaque période considérée, ne soit différent d'un lot à l'autre.

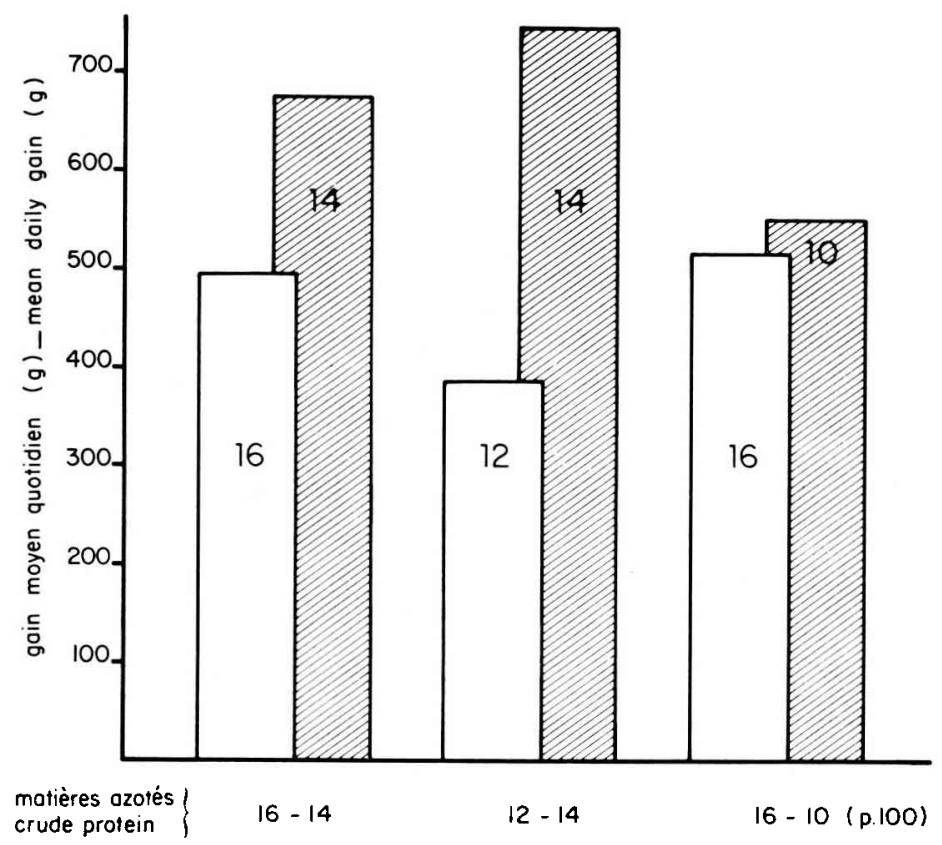

FIG. I. - Croissance journalière moyenne entre 28 et $100 \mathrm{~kg}$ de poids vif. Mean daily gain between 28 and $100 \mathrm{~kg}$ live-weight

$\square$ entre 28 et $60 \mathrm{~kg}$ de poids vif (between 28 and $60 \mathrm{~kg}$ live weight).

entre 60 et $100 \mathrm{~kg}$ de poids vif (between 60 and Ioo $\mathrm{kg}$ live-weight). 
Avant $60 \mathrm{~kg}$ de poids vif (niveau moyen de consommation : $\mathrm{I}, 56 \mathrm{~kg}$ matière sèche par jour), l'abaissement du taux protéique de I6 à I2 p. Ioo diminue la croissance journalière des animaux d'environ 23 p. Ioo et accroît, parallèlement, l'indice de consommation de $30 \mathrm{p}$. roo. Après $60 \mathrm{~kg}$ de poids vif et jusqu'au stade de Ioo $\mathrm{kg}$ pour les animaux non pubères à ce poids (consommation moyenne : $2,42 \mathrm{~kg}$ matière sèche par jour), l'abaissement du taux de matières azotées de I4 à Io p. Ioo provoque également un ralentissement de la croissance d'environ I9 p. Ioo et une augmentation de l'indice de consommation de $27 \mathrm{p}$. Ioo. Ainsi, sur la période totale considérée (de 28 à Ioo $\mathrm{kg}$ de poiđs vif), les restrictions protéiques imposées induisent une diminution significative de la vitesse de croissance des animaux de 8 à II p. IOO.

\section{2. - Age et poids à la puberté}

Le ralentissement de la croissance observé dans les deux groupes d'animaux restreints en matières azotées, avant ou après $60 \mathrm{~kg}$ de poids vif, ne se répercute que faiblement sur le déclenchement de la puberté. Le retard, non significatif,

\section{TABLEAU 2}

Influence de l'apport azoté à partiv de $28 \mathrm{~kg}$ de poids vif sur la maturité sexuelle et sur le taux d'ovulation au premier astrus Effect of protein intake after $28 \mathrm{~kg}$ live-weight on puberty and ovulation rate

\begin{tabular}{|c|c|c|c|c|}
\hline 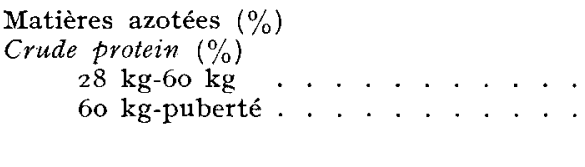 & $\begin{array}{l}16 \\
14\end{array}$ & $\begin{array}{l}\text { I2 } \\
\text { I } 4\end{array}$ & $\begin{array}{l}\text { I6 } \\
\text { I0 }\end{array}$ & $\begin{array}{l}\text { Signification } \\
\text { statistique } \\
\text { Statistical } \\
\text { significance } \\
\quad \mathrm{Sx}()^{1}\end{array}$ \\
\hline Nombre de truies (Number of gilts) . . . . . & 29 & 34 & 30 & \\
\hline 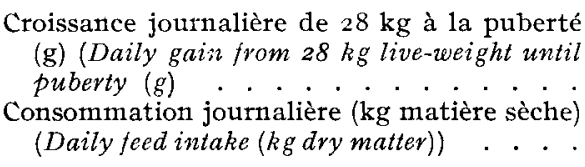 & $\begin{array}{l}585 a \\
2,00\end{array}$ & $\begin{array}{l}535 b \\
I, 96\end{array}$ & $\begin{array}{l}519 b \\
2,08\end{array}$ & $\begin{array}{l}\text { I I }(\mathrm{I} 2,0) * * * \\
0,03(\mathrm{r} 0,0) \mathrm{NS}\end{array}$ \\
\hline 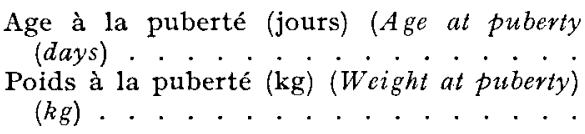 & $\begin{array}{l}240,2 \\
\text { I I } 7,0\end{array}$ & $\begin{array}{l}249, \mathrm{I} \\
\text { I I } 4,6^{2}\end{array}$ & $\begin{array}{l}249,0 \\
\text { I I I }, 2\end{array}$ & $\begin{array}{l}4,4(10,4) \mathrm{NS} \\
2,9\left(\mathrm{I}_{4}, 8\right) \mathrm{NS}\end{array}$ \\
\hline Taux d'ovulation (Ovulation rate) . . . . . & $\mathrm{r}_{3}, 8$ & I $3, \mathrm{I}$ & I 2,7 & $0,4 \quad(16,4) \mathrm{NS}$ \\
\hline
\end{tabular}

(1) Ecart-type de la moyenne (cofficient de variation). T,es valeurs suivies de la même lettre ne diffèrent pas entre elles. NS : différence non significative; * différence significative au seuil $\mathrm{P}<0,05 ; * * \mathrm{P}<0,01$; $* * * \mathbf{P}<0,001$. (Standard error of the mean (cofficient of variation): The mean values followed by the same letter are not significant. NS: non significant; Levels of significance* $0.05 ; * * 0.01 ; * * * 0.001$. 
observé, est en effet de 9 jours (tab1. 2). De la même façon, le poids des animaux à ce stade ne diffère pas dans les 3 lots. Cependant, on peut noter une tendance à l'augmentation du taux d'ovulation au premier nestrus, dans le lot témoin, en regard des moyennes enregistrées dans les lots 2 ou 3, l'écart maximum entre lots n'étant toutefois que de I,I ovules pondus.

\section{3. - Performances de reproduction à 30 et à I05 jours de gestation}

Au cours de leur gestation, les animaux subissent l'effet rémanent du régime prépubertaire et l'effet du taux protéique du régime de gestation. Au cours du premier mois de gestation (tabl. 3), la croissance maternelle est comparable dans les différents lots ( 545 grammes par jour), si l'on excepte le groupe de truies bénéficiant, après une restriction protéique entre $60 \mathrm{~kg}$ de poids vif et la puberté, d'un accroissement du taux de matières azotées dans le régime de gestation $(645$ grammes par jour). Si l'on considère la presque totalité de la gestation (tabl. 4), on peut noter que les truies restreintes pendant la période prépubertaire (lots 2 et 3) présentent une croissance légèrement plus rapide durant leur gestation ( 478 contre 43 I grammes par jour). Par ailleurs, durant cette période, le gain net maternel, déduit du gain brut de gestation après prise en compte du poids utérin, est accru, significativement, au taux protéique le plus élevé $(39,0$ contre $34,4 \mathrm{~kg})$.

Pour ce qui concerne le nombre d'embryons ou de fotus, il n'apparait pas de différences significatives d'un lot à 1'autre. Cependant, on peut constater une tendance à l'augmentation de la mortalité in utero, d'une part quand le taux d'ovulation est accru (cas du lot témoin) d'autre part, quand les animaux reçoivent en gestation l'aliment à taux protéique le plus élevé. Dans ce dernier cas, le poids moyen des foetus est plus grand ( $956 \mathrm{~g}$ contre 9o8) mais cette supériorité pondérale n'est pas totalement expliquée par une prolificité moindre. On mentionnera également une diminution significative du poids moyen des foetus (852 $\mathrm{g}$ contre 963), provenant de mères non restreintes en protéines avant la puberté.

Pour ce qui concerne enfin les poids moyens des membranes placentaires, aux deux stades considérés, si certaines différences entre lots apparaissent significatives, l'origine de ces différences reste imprécisée.

\section{4. - Caractéristiques des carcasses}

Différents facteurs peuvent modifier les caractéristiques de la composition corporelle des animaux abattus. On retiendra essentiellement les facteurs inhérents au schéma expérimental, c'est-à-dire d'une part le taux protéique du régime alimentaire avant ou après saillie, d'autre part, le stade de gestation. L'ensemble des résultats est consigné dans le tableau 5. Compte tenu d'un poids moyen des animaux différent dans chaque lot et à chaque stade d'abattage, on a exprimé les principaux paramètres de la composition corporelle en pourcentage du poids net (poids de la carcasse après un ressuyage de 24 heures).

L'effet du régime prépubertaire est surtout marquant au stade le plus précoce de la gestation. Ainsi, les animaux recevant la séquence azotée optimale durant leur croissance ( $\left.6 / \mathrm{I}_{4}\right)$ ont tendance, pour un poids vif d'abattage comparable, à avoir un meilleur degré de musculature (pourcentage de longe et de jambon) que les animaux subissant une restriction protéique. Inversement, dans ce dernier cas, les pourcentages de morceaux gras (bardière ou panne) sont accrus et ceci 


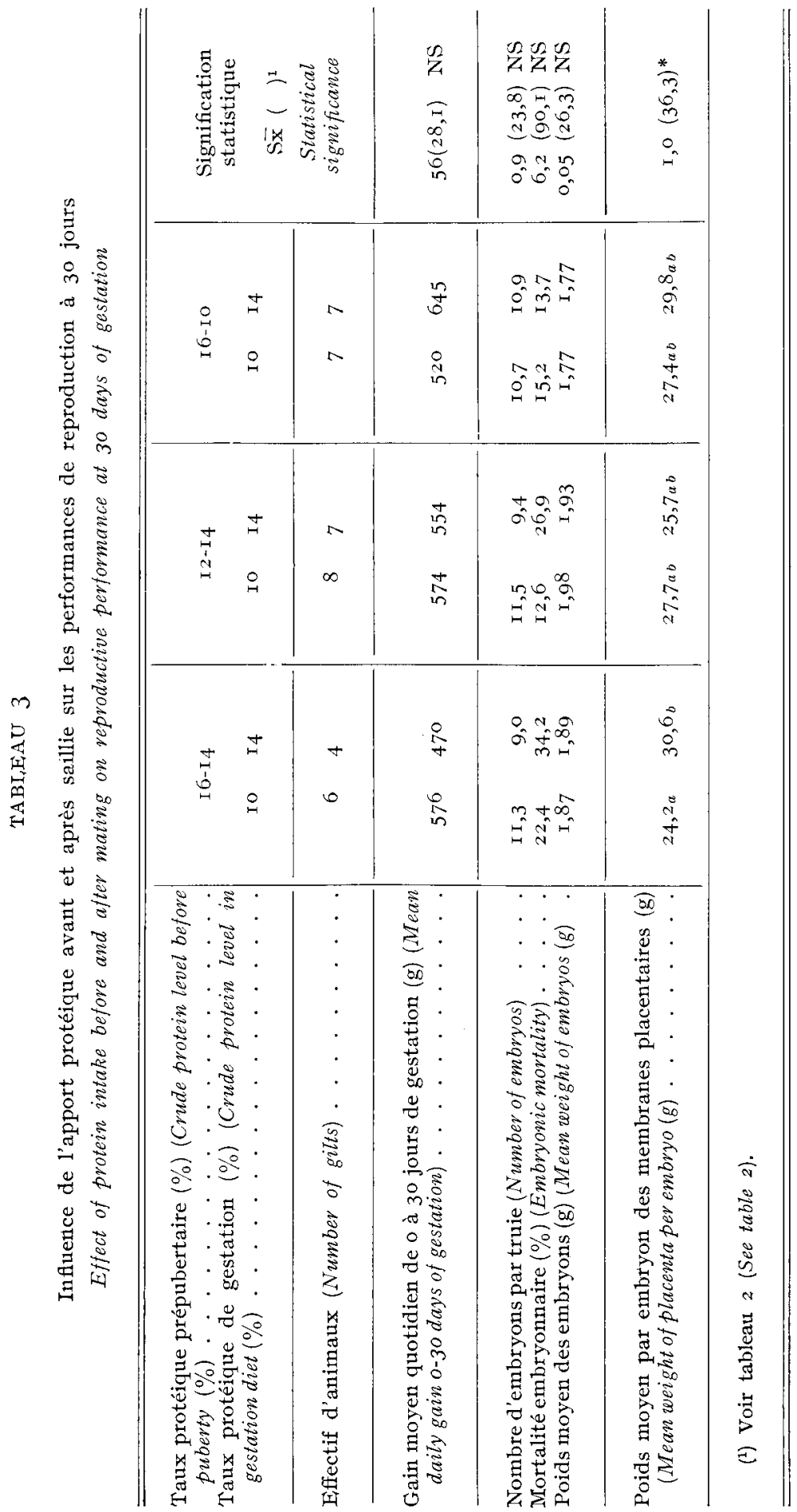




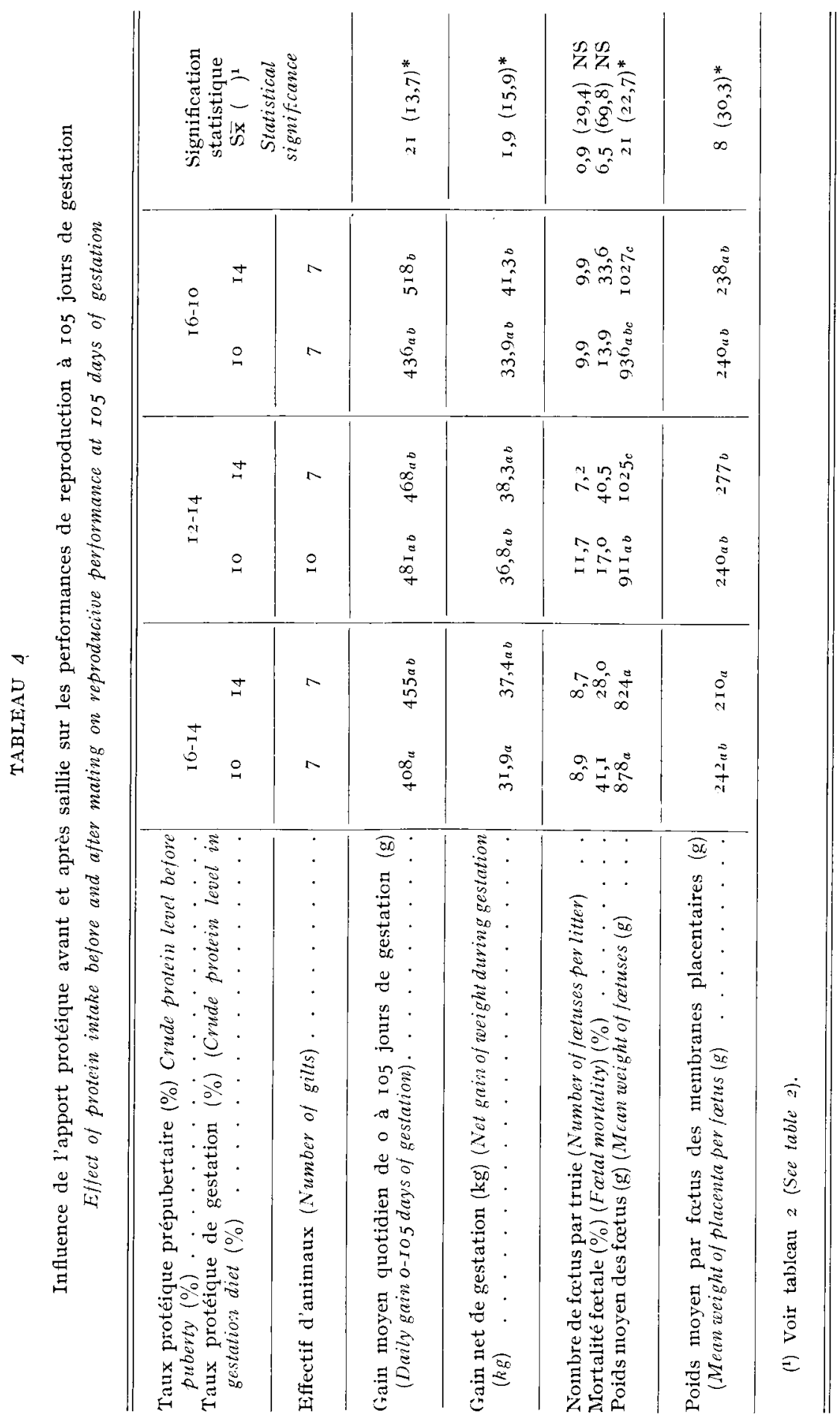


APPORT AZO'TÉ E'T REPRODUCTION CHIZ LA TRUIE
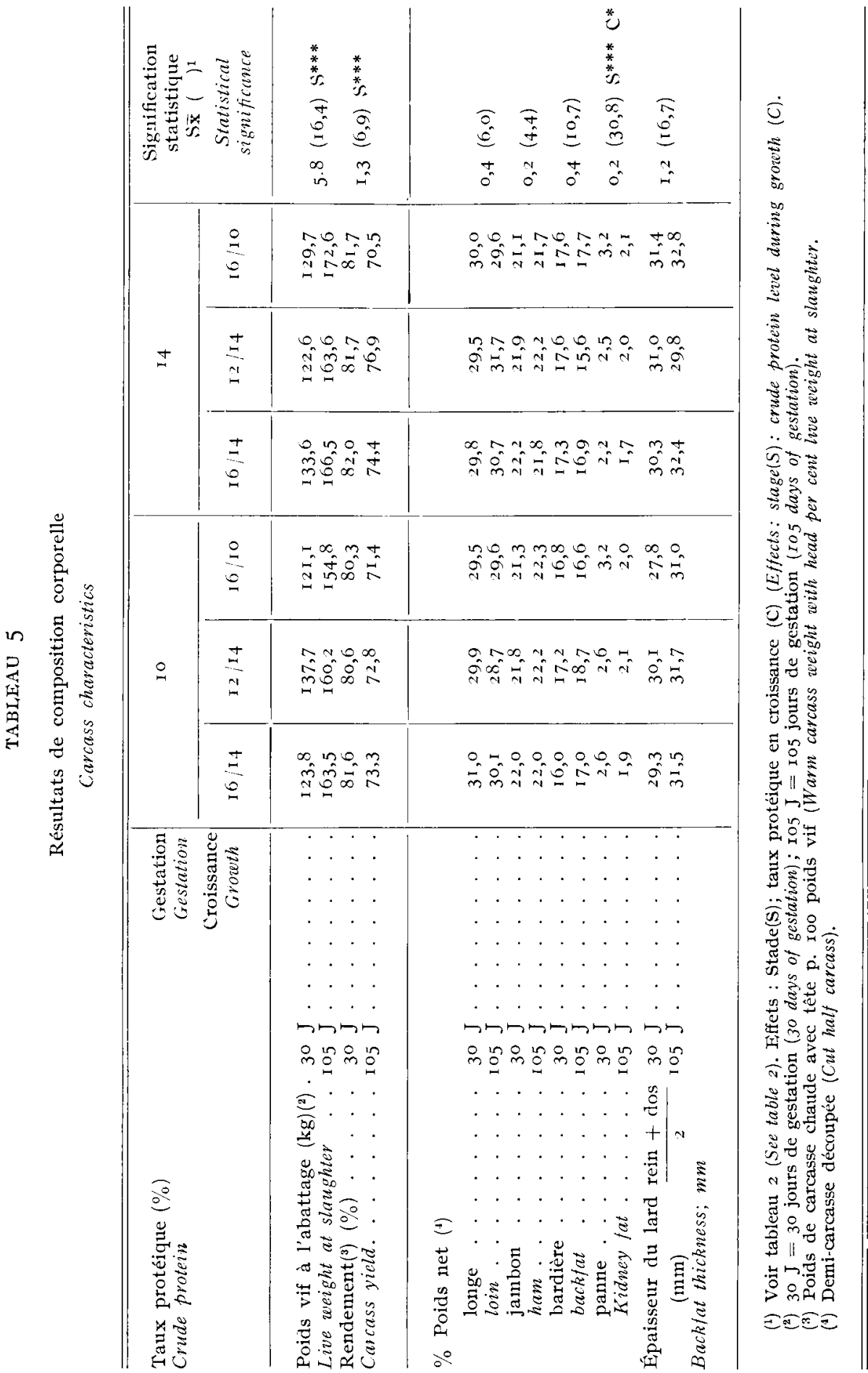
est particulièrement visible pour le gras périrénal quand la restriction protéique est appliquée à partir de $60 \mathrm{~kg}$ de poids vif (augmentation significative de près de 20 p. IOO).

Pour ce qui concerne l'effet du régime de gestation, les différences de composition corporelle, quoique non significatives, sont surtout mises en évidence au stade le plus tardif de la gestation. Ainsi, l'augmentation du taux protéique de gestation accroît le poids vif à l'abattage, de $7,8 \mathrm{~kg}$ en moyenne, ainsi que le rendement d'abattage de I,4 points (tab1. 5). Par ailleurs, le pourcentage de longe passe, en moyenne, de 29,4 p. I oo à 30,7 p. Ioo, augmentation compensée principalement par une diminution du gras dorsal (de I7,6 p. Ioo à I $6,7 \mathrm{p}$. roo). Cette tendance générale n'est cependant pas reflétée par les animaux de la séquence prépubertaire I6/Io; à cet égard, il faut remarquer, dans ce lot, l'écart relativement important du poids vif à l'abattage à Io $\mathrm{p}$. Ioo de protéines ( $\mathrm{I} 54,8 \mathrm{~kg}$ ) et à I 4 p. Ioo $(\mathrm{I} 72,6$ $\mathrm{kg})$.

Enfin, on constate un effet très significatif du stade de gestation sur le rendement à l'abattage qui passe de 8 I, 2 p. Ioo à 30 jours à 73,3 p. IOO à IO5 jours. La composition du poids net de carcasse semble relativement constante au cours de la gestation; seul, le pourcentage de gras périrénal diminue d'environ 30 p. Ioo en fin de gestation.

Ces données d'analyse corporelle perniettent une approche de la composition du gain net des truies entre 30 et I05 jours de gestation, suivant le taux protéique du régime (tab1. 6). Les quantités de morceaux nobles (longe et jambon) et de

\section{TABI EAU 6}

Fivolution du poids des morceanx an cours de la gestation

lean and fat cuts during gestation

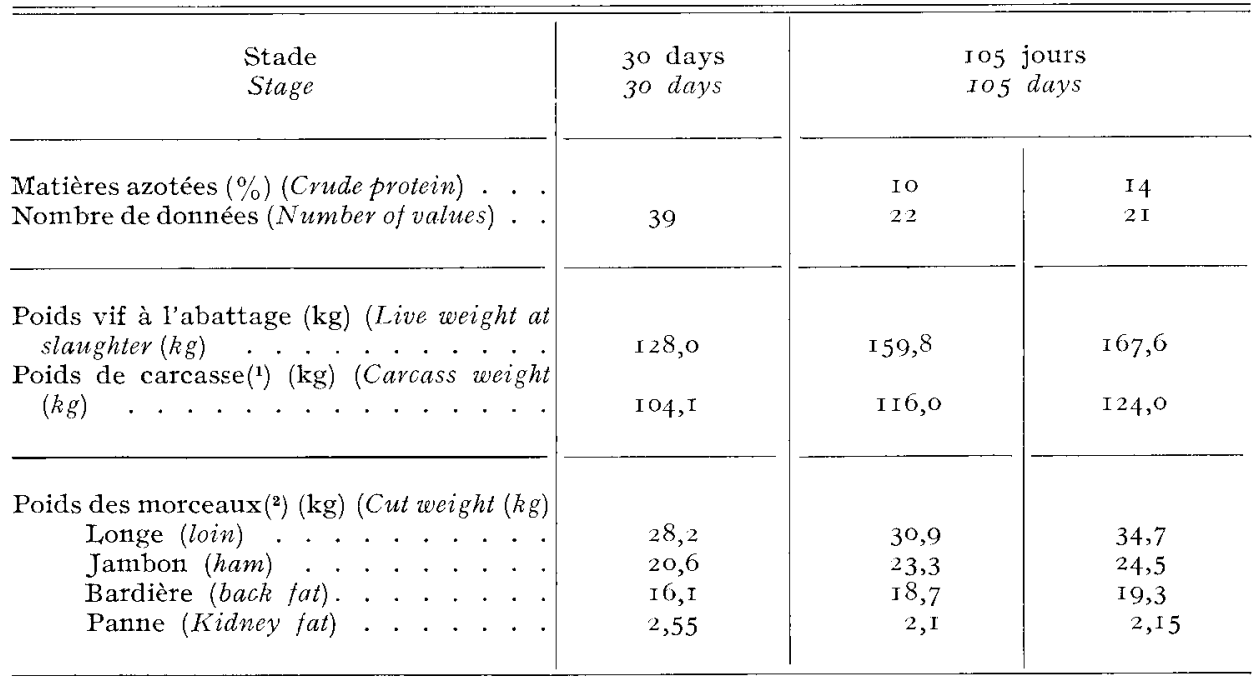

(1) Poids de carcasse chaude avec tête (Warm carcass weight with head).

(2) A partir de la composition de la demi-carcasse découpée, 24 heures après l'abattage (From the composition of cut half-carcass, 24 hours after slaughter). 


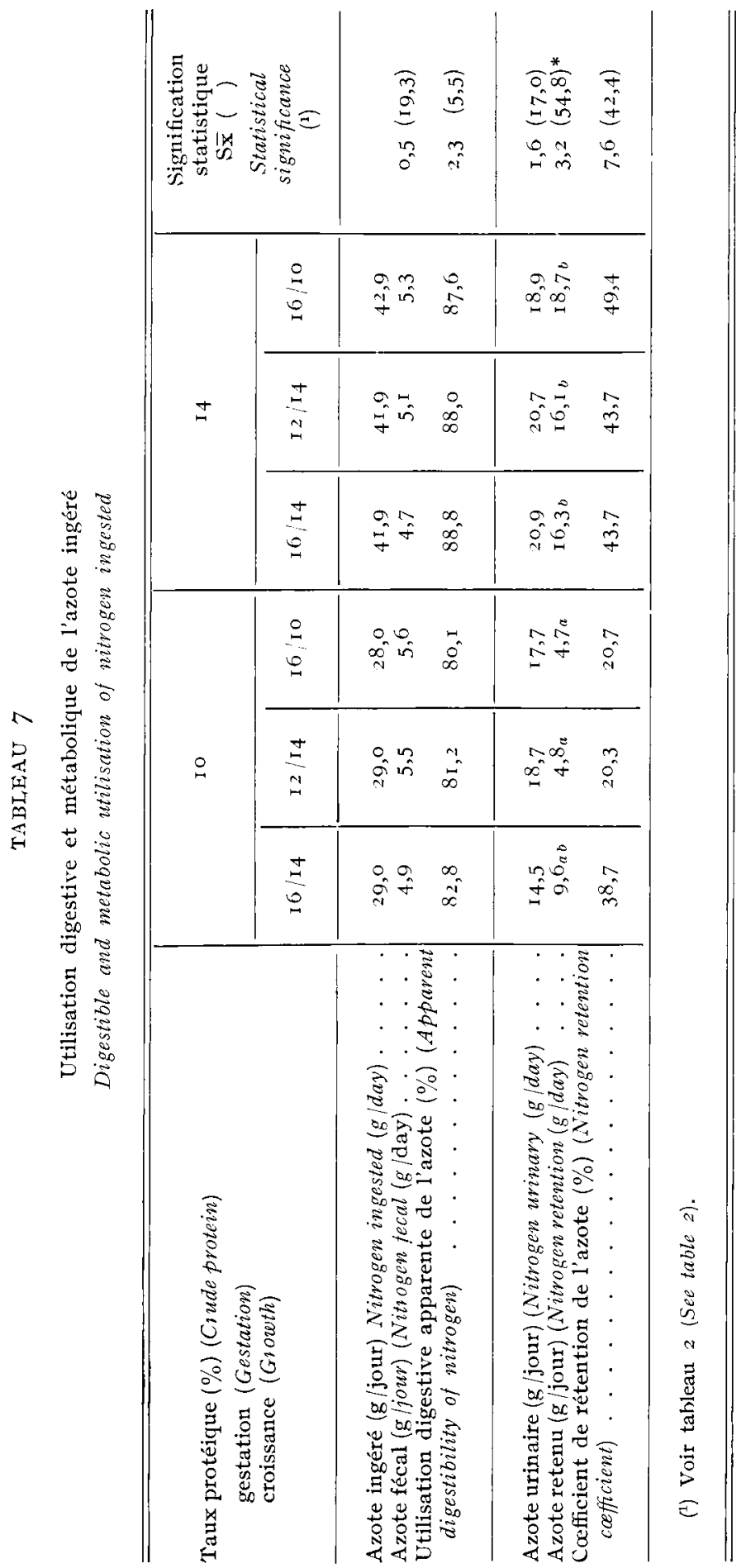


bardière augmentent au cours de la gestation; seul, le gras périrénal diminue en valeur absolue. A ro p. Ioo de protéines dans la ration, le gain de carcasse (mesuré à chaud, avec tête) est de II,9 kg dont près de $70 \mathrm{p}$. Ioo peuvent être répartis équitablement entre la longe, le jambon et la bardière. Par contre, à I4 p. Ioo de protéines, le gain net de carcasse est de $19,9 \mathrm{~kg}$, comprenant un pourcentage beaucoup plus important de longe ( 33 p. IOo), en piace du gras dorsal ( 6 p. IOO). Ainsi, pour la période d'observation considérée, le rapport entre les gains respectifs de longe et de bardière est voisin de l'unité au taux protéique le plus bas et est doublé au taux prốéique le plus élevé.

\section{5. -- Utilisations digestive et métabolique de l'azote ingéré}

L'étude en cages à métabolisme, réalisée vers le $80^{\mathrm{e}}$ jour de gestation porte sur un nombre de truies correspondant à $53 \mathrm{p}$. Ioo de 1'effectif abattu à 105 jours de gestation. Les résultats sont consignés dans le tableau 7 .

Les pertes azotées fécales sont con:parables d'un lot à l'autre, ce qui se traduit par une amélioration du coefficient d'utilisation digestive apparente au taux azoté le plus élevé. A ce niveau de consommation azotée et maígré des pertes azotées urinaires accrues, le bilan azoté est également amélioré, Toutefois, ce bénéfice est moins marqué chez les animaux non restreints avant la puberté. D'ailleurs, pour ceux-ci, l'accroissement de la consommation azotée en gestation ne modifie pas le coefficient de rétention de l'azote.

En définitive, cette étude montre qu'un surcroît de consommation azotée en gestation permet une amélioration de la rétention d'azote, dont l'ampleur dépend de l'apjort protéique prépubertaire.

\section{Discussion et conclusions}

\section{I. -- Effet du taux protéique avant la puberté}

L'abaissement du taux global de matières azotées dans la ration, à partir de $28 \mathrm{~kg}$ de poids vif, induit une diminution sensible de la vitesse de croissance et une augmentation de l'indice de consommation, quel que soit le stade d'application, et ceci a été maintes fois observé, particulièrement chez le porc femelle (HENRY, I980). Cette relation aboutit à l'obtention de profils de croissance différents, en fonction de la chronologie des apports protéiques. L'effet sur 1'âge au premier cstrus est cependant minime. Il s'avère ainsi que dans les lots restreints (avant ou après $60 \mathrm{~kg}$ de poids vif) le retard non significatif à la puberté est de 9 jours, écart comparable à celui observé antérieurenient (DUEE, I977) ou par Jones et MAXWELL (I974). I, a diminution des apports protéiques, tardivement (à partir de $65 \mathrm{~kg}$ de poids vif) et pour une souche de porcs à maturité sexuelle peu précoce, peut même être sans effet (WIESEMÜLI,ER, GünThER et PILs, I976). Par contre, si la restriction protéique est appliquée durant ure grande partie de la croissance, le retard à la puberté peut atteindre i 9 à 26 jours (CunNinginam et al., I974; WAHISTROM et LiBAI, I977). On peut donc admettre, à travers les résultats de cette étude, qu'une restriction protéique limitée à une partie de la croissance n'a que peu d'influence sur l'âge à la puberté, même si la vitesse de croissance est modifiée : l'absence de corrélation significative entre ces deux 
paramètres traduit cet état de fait. I)'ailleurs, selon FRIEND (I976), l'apport énergétique, plus que 1'apport protéique, affecte l'âge au premier œstrus. Toutefois, d'autres facteurs peuvent être mis en cause. Si 1'on rapproche les résultats obtenus dans l'expérience présente des données enregistrées par BOLET, ETIENNE et LEGAULT (I977), il ressort que certaines conditions d'élevage sont susceptibles de modifier 1'âge à la puberté, probablement par le biais de la vitesse de croissance des animaux. Ainsi, un groupe de jeunes femelles de même origine génétique et élevées dans des conditions comparables à celles du lot témoin (alimentation équilibrée, bâtiment fermé) présentent un âge à la puberté de 235 jours (240 jours dans l'étude présente), mais qui peut être abaissé de 2 I jours dans d'autres conditions d'ambiance (porcheries de type semi plein air). Parallèlement, la vitesse de croissance des animaux est améliorée $(+\mathrm{I} 78 \mathrm{~g} / \mathrm{j})$. On peut alors se demander si pour des porcs à forte potentialité de croissance ou élevés dans des conditions qui leur permettent d'extérioriser leurs potentialités de croissance, une restriction protéique, même passagère, ne pourrait pas retarder l'apparition de la maturité sexuelle.

Malgré un âge à la puberté plus élevé, on note un poids plus faible à ce stade pour les animaux restreints en protéines, et ceci est à rapprocher d'une diminution du taux d'ovulation. D'ailleurs, une relation linéaire significative existe entre ces deux paramètres $(\gamma=0,23)$, ce qui a déjà été souligné (E'TIENNE et DUEE, I973; DUEF, I977). Toutefois, cette diminution de la taille de portée potentielle ne se répercute pas sur le nombre d'embryons à 30 jours ou de foetus à ro5 jours. En effet, la mortalité in utéro, aux deux stades, apparaît légèrement plus élevée chez les animaux témoins (27, I p. Ioo contre I6,8 p. Ioo à 30 iours; 34 , I p. Ioo contre 25,0 p. IO0 à I05 jours). Cette régulation de l'effectif de la population tutérine à un seuil caractéristique de la souche utilisée a été montrée par ailleurs (BAZER et al., rg69) et est plus particulièrement rencontrée chez la nullipare. Si elle traduit l'impossibilité d'obtenir une taille de portée supérieure à une "limite physiologique ", elle n'exclut pas cependant l'éventualité de sa diminution, quand le taux d'ovulation est trop abaissé (ETIENNE et DUEE, I973).

En définitive, dans les conditions expérimentales précisées antérieurement, on peut conclure qu'une restriction protéique de 25 à $40 \mathrm{p}$. Ioo selon le stade d'application et limitée à une partie de la croissance ne porte pas préjudice aux performances ultérieures de reproduction (âge à la puberté, taille de portée en première gestation).

\section{2. - Effet du taux protéique en première gestation}

L'influence minime de l'apport protéique durant la gestation sur les performances de reproduction ne doit pas surprendre. On sait, après les expériences de Pond et al. (I968) que le développement fotal (nombre et poids des porcelets à la naissance) supporte des régimes carencés en protéines. Toutefois, il existe une tendance à 1'accroissement de la mortalité in utéro avec le taux protéique le plus élevé, qui se traduit par une diminution non significative du nombre de fœetus en fin de gestation avec le régime à $14 \mathrm{p}$. roo de protéines, tendance déjà rencontrée (DUEE, I976). Cependant, GREENHALGH et al. (I977) ont montré 1'effet inverse et cette contradiction apparente n'est que le reflet de la grande variabilité du paramètre considéré. On peut également admettre que 1'augmentation significative du poids moyen des fotus avec le régime le plus riche en protéines est d'abord une conséquence de la diminution de la taille de la portée. 
L'influence de l'apport protéique durant la gestation est plus marquée sur le gain de poids maternel. Ainsi la diminution du taux protéique de $\mathrm{I}_{4}$ à Io $\mathrm{p}$. roo a pour effet de diminuer le gain de poids des animaux et ceci est bien mis en évidence au stade ultime de l'étude : le gain net maternel est alors abaissé de i2 p. Ioo comme l'ont déjà montré Holdex et $a l$. (I968), Hawtoñ et MEADE (I97I). Parallèlement, la quantité d'azote retenu en fin de gestation est réduite (de $\mathrm{I} 7,0$ à $6,4 \mathrm{~g}$ par jour), ce qui correspond principalement à une quantité moindre de masses musculaires déposées si l'on se réfère aux résultats de découpe de la carcasse (DEsmoulin, Grandsart et TAssencourt, I976).

On sait que l'état de gestation, comparé à l'état des animaux non gravides, se traduit par un dépôt musculaire accru, illustré par un niveau plus élevé de rétention azotée (SAIMON-LEGAGNEUR, I965; ElLSLEy et al., I 966 ; HFAP et LODGE, I 667). Inversement, les masses adipeuses dans la carcasse régressent, ce qui est démontré dans l'expérience présente par l'évolution du pourcentage de gras périrénal.

Cette modification de la composition corporelle au cours de la gestation, permettant de ce fait l'obtention de carcasses plus maigres, semble conditionnée par l'apport protéique durant cette période mais également avant la puberté. Fin effet, il apparaît qu'au taux protéique le plus bas en gestation, seuls les animaux ayant reçu le régime témoin avant la puberté réalisent un dépôt azoté élevé, associé à un meilleur degré de musculature. L,a restriction azotée prépubertaire, surtout après $60 \mathrm{~kg}$ de poids vif, si elle permet des gains de poids plus élevés en gestation, n'aboutit cependant pas à l'obtention de carcasses plus maigres en fin de gestation. Dans ce dernier cas, une tendance comparable est perçue même à $\mathrm{I} 4 \mathrm{p}$. Ioo de protéines dans le régime et ces résultats rejoignent les conclusions d'HOWEI, et al. (I977 $a$ et $b$ ) qui soulignaient l'influence du poids à la saillie sur les modifications de la composition corporelle au cours de la gestation.

En conclusion, il ressort de cette étude qu'une réduction de l'apport azoté durant une partie de la croissance modifie davantage l'évolution de la composition corporelle au cours de la première gestation que les paramètres de reproduction (âge à la puberté, prolificité). La faculté pour la truie gestante de mieux valoriser l'apport protéique semble dépendante d'une part du niveau de cet apport, d'autre part de l'état nutritionnel des animaux au début de la gestation. On souligne, par ce biais, l'interaction qui existe entre ces deux périodes, la période prépubertaire et la première gestation, et la nécessité d'une meilleure connaissance de l'anabolisme vrai de gestation en fonction des apports nutritionnels, compte tenu de son rôle essentiel au cours de la lactation suivante.

Accepté pour publication en mai ig8o.

\section{Summary} Influence of protein supply during growth and first gestation
on reproductive performance and body composition of the gilt

An experiment was carried out on r 20 Large White gilts from an average live-weight of $20 \mathrm{~kg}$ and until term of the first gestation. Until puberty, the animals were distributed into three groups, only differing by the crude protein level of the diet offered. A control group (group I : i6 p. Ioo crude protein until $60 \mathrm{~kg}$ live-weight, and then I4 p. roo) was used to compare the effect on growth and reproductive performance of a protein level reduction before $60 \mathrm{~kg}$ live-weight (group $2:$ I 2 p. IOO) or after $60 \mathrm{~kg}$ live-weight (group $3:$ IO p. IOo). The level of 
feed intake was the same for all the animals and changed according to the weight until a plateau of $2.8 \mathrm{~kg}$ per day (reached at $80 \mathrm{~kg}$ live-weight). The protein sources were maize supplemented with soyabean oil meal. At puberty, the gilts received one of the two diets of the finishing period ( 14 or Io p. Ioo protein), at the same feeding level, i.e. $2 \mathrm{~kg}$ per day until 30 days and then I. $8 \mathrm{~kg}$.

Half of the animals in each of the six groups was slaughtered towards day 30 of gestation; the other half towards day ro5. The protein restriction during a part of the growing period reduced the growth rate of animals (between $28 \mathrm{~kg}$ live-weight and puberty) from $8 \mathrm{p}$. I oo (group 2) to I I p. Ioo (group 3), but the non significant delay at puberty was only nine days (group I : 240 days; groups 2 and $3: 249$ days), with a low ovulation rate (group I : I3,8; group $2:$ I3.I; group $3: 12,7$.

There was no significant difference between groups in the number of embryos (30 days) or fotuses (I05 days) : the mortality in utero being higher in the control group.

The influence of the protein supply during gestation was mainly marked in the dam. Decrease in the protein level from I4 to Io p. Ioo reduced the net weight gain of the animals by I 2 p. I oo, as well as the nitrogen retention during late gestation (from 17.0 to $6.4 \mathrm{~g}$ per day), corresponding to a smaller muscle deposition, the magnitude of which was estimated by the proportions of loin and ham in the carcass.

However, it seems that the protein supply before puberty affects the changes in the body composition during gestation. A protein restriction during growth followed by the lowest protein level during gestation reduces the nitrogen retention and leads to a lower muscle deposition than that obtained in the control group in spite of the higher growth during gestation.

\section{Références bibliographiques}

Bazer F. W., Robison O. W., Clawson A. J., UlberG. L. C., 1969. Uterine capacity at two stages of gestation in gilts following embryo superinduction. J. Anim. Sci., 29, 30-34.

BOLET G., ETIENNE M., LEGAULT C., I977. Effets de l'habitat et de l'origine génétique sur les performances d'engraissement et la précocité sexuelle de jeunes truies de race Irarge White. Ann. Zootech., 26, 255-27r.

Carrez S., Treil F., Duee P. I., Aumaitre A., I977. Influences sur la puberté de la truie de sa période de naissance et de sa durée d'allaitement. Ann. Zootech., 26, 62 I-625.

Cunningham P. J., NABER C. H., Zimmerman D. R., PEo E. R., Jr., i974. Influence of nutritional regime on age at puberty in gilts. J. Anim. Sci., 39, 63-67.

Desmoulin B., Grandsart P., TAssencourt L., i 976 . Les critères d'appréciation de la composition anatomique de la carcasse du porc et des pièces de découpe. Journées Rech. porcine en France, 8, 89-98. I.N.R.A. - I.T.P. éd., Paris.

DuEE P. H., 1976. Chronologie de l'apport azoté pendant le cycle de reproduction chez la truie. Ann. Zootech., 25, I99-212.

DUEE P. H., I977. Influence d'une restriction azotée, à partir de $25 \mathrm{~kg}$ de poids vif, sur les performances de reproduction de la truie. Journées Rech. porcine en France, 9, I93-I98. I.N.R.A.I.T.P. éd., Paris.

Elshey F. W. H., Anderson D. M., McDonaid I., MacPherson R. M., SMar'T R., I966. A comparison of the live-weight changes, nitrogen retention and carcass composition of pregnant and non-pregnant gilts. Anim. Prod. 8, 391-400.

Fitienne M., DUEE P. H., I 973. Effets respectifs des niveaux alimentaires pendant la croissance et le premier mois de gestation sur les performances de reproduction chez la truie nullipare : résultats préliminaires. Ann. Zootech., 22, 453-462.

FrIFND D. W., I976. Nutritional effects on age at puberty and plasma amino acid level in Yorkshire gilts and on chemical composition, nucleic acid, fatty acid and hydroxyproline contents of the uterus. $J$. Anim. Sci., 43, 404-4I2.

Greenhalgh J. F. D., Elsley F. W. H., Grubb D. A., Lightroot A. L., Saul D. W., Smith P., WAlker N., Wililiams D., YeO M. L., I977. Coordinated trials on the protein requirements of sows. I- A comparison of four levels of dietary protein in gestation and two in lactation. Anim. Prod., 24, 307-321.

HAWTON J. D., MEADE R. J., I97I. Influence of quantity and quality of protein fed the gravid female on reproductive performance and development of offspring in swine. $J$. Anim. Sci., $32,88-95$ 
HEAP F. C., LODGE G. A., I967. Changes in body composition of the sow during pregnancy. Anim. Prod., 9, 237-245.

HeNRY Y., PION R., Rerat A., I976. Protein supply for pigs and possibilities of reducing protein feeding standards. World. Rev. Anim. Prod., 12, 9-32.

HENRY Y., I980. Besoin azoté global du porc en croissance : Résultats sur femelles et synthèse. Journées Rech. porcine en France, 12, 183-194. I.N.R.A. - I.T.P. éd., Paris.

Holden P. J., Lucas E. W., Speer V. C., HAYs V.W., ig68. Effect of protein level during pregnancy and lactation on reproductive performance of sows. $J$ Anim. Sci., 27, I587-I 590 .

Howeli, F. D. B., MacPhifrson R. M., Crofts R. M. J., Pennie K., I977a. The effect of energy intake and mating weight on growth, carcass yield and litter size of female pigs. Anim. Prod., 25, 233-245.

Howeit F. D. B., MacPherson L. M., Crofts R. M. J., Smart R. I., I977b. The effect of pregnancy, energy intake and mating weight on protein deposition and energy retention of female pigs. Anim. Prod., 25, 281-29o.

JONES R. D., MAXWEI, C. V., I974. Effect of protein level on growth, nitrogen balance and reproductive performance in gilts. J. Anim. Sci, 39, 1067-1072.

LODGE G. A., MacPherson R. M., I96I. Level of feeding during early life and the subsequent reproductive performance of sows. Anim. Prod., 3, 19-28.

Pond W. G., Wagner W. C., Dunn J. A., Walker F, F., Ig68. Reproduction and early postnatal growth of progeny in swine fed a protein-free diet during gestation. J. Nuir., 94, 309-316.

RERAT A., DUEE P. H., 1976. Conditions d'élevage et alinentation chez la jeune truie : influence sur la carrière ultérieure de l'animal in La conduite du troupeau de reproducteurs porcins, édité par Etudes et Formation Vétérinaires, I5-24.

SALMON-LEGAGNEUR E., I965. Quelques aspects des relations nutritionnelles entre la gestation et la lactation chez la truie. Ann. Zootech., 14, nº H.S., I37.

WALHSTROM R. C., IIBAL, G. W., I977. Effect of dietary protein during growth anci gestation on development and reproductive performance of gilts. J. Anim. Sci., 45, 94-99.

WIESEMÜllfer W., Günther K., Pils H., 1976. Untersuchungen über den Einfluss der Proteinernährung von Jungsauen während der Aufsucht sowie in den folgenden Graviditäten und Laktationen auf die Fortpflanzungsleistungen. Tievernähr. Fütterung, 10, 136-142. 